\title{
A Rich Example of Geometrically Induced Nonlinearity: From Rotobreathers and Kinks to Moving Localized Modes and Resonant Energy Transfer
}

\author{
P. G. Kevrekidis ${ }^{1}$, S. V. Dmitriev ${ }^{2,3}$, S. Takeno ${ }^{4}$, A. R. Bishop ${ }^{5}$ and E. C. Aifantis ${ }^{6}$ \\ ${ }^{1}$ Department of Mathematics and Statistics, \\ University of Massachusetts Lederle Graduate Research Tower, \\ Amherst, MA 01003-4515, USA \\ ${ }^{2}$ Institute of Industrial Science, \\ the University of Tokyo, 4-6-1 Komaba, \\ Meguro-ku, Tokyo 153-8505, Japan \\ ${ }^{3}$ National Institute of Materials Science, \\ 1-2-1 Sengen, Tsukuba, Ibaraki 305-0047, Japan \\ ${ }^{4}$ Graduate School, Nagasaki Institute of Applied Science, \\ Nagasaki 851-0193, Japan \\ ${ }^{5}$ Center for Nonlinear Studies and Theoretical Division, \\ Los Alamos National Laboratory, \\ Los Alamos, NM 87545 USA \\ ${ }^{6}$ Laboratory of Mechanics and Materials, \\ Aristotle University of Thessaloniki, \\ GR 54124, Thessaloniki, Greece
}

(Dated: November 16, 2018)

\begin{abstract}
We present an experimentally realizable, simple mechanical system with linear interactions whose geometric nature leads to nontrivial, nonlinear dynamical equations. The equations of motion are derived and their ground state structures are analyzed. Selective "static" features of the model are examined in the context of nonlinear waves including rotobreathers and kink-like solitary waves. We also explore "dynamic" features of the model concerning the resonant transfer of energy and the role of moving intrinsic localized modes in the process.

PACS numbers: 05.45.-a, 05.45.Yv, 63.20.-e
\end{abstract}

\section{INTRODUCTION.}

In the past few years, there has been a dramatic increase of interest in the behavior of solitary waves and intrinsic localized modes (ILMs) in dynamical lattices; see e.g., 1] for a number of recent reviews. One of the key reasons for this focus of interest has been the ability of such modes (which are ubiquitous in nonlinear lattice models) to localize the energy and transfer it in a targeted way [2, 3]. An additional, related feature of these modes is the important role they play in the conduction of heat (or equivalently transport of energy) along such dynamical simple lattices and how this relates to fundamental macroscopic laws of thermodynamics such as Fourier's law of heat conduction; see e.g., [4] for a recent review. Another application may be found in modelling the deformation and fracture behaviour of continuous structured media with internal degrees of freedom. Some initial model equations in this direction can be found in [5].

On the other hand, an increasingly important theme in nonlinear physics concerns the interplay between nonlinear dynamics and geometry, especially in lattice settings. The relevant contexts vary from long-range interactions on a fixed curved substrate [6], to substratefeedback models [7] and coupled atomic chains [8], and from junctions between lattices with different masses 9 to semi-circular polymer-like chains [10] and models of geometrically nontrivial DNA 11]. The unifying principle in all these situations is that the geometry can significantly affect the static properties of excitations in the lattice (e.g., multi-stability), as well as dynamical ones (e.g., a variety of outcomes in the interaction of intrinsic localized modes with curvature).

Motivated by these two emerging themes of nonlinear lattice dynamical systems, we propose in this work a mechanical example, in which even though the underlying interactions of the system consist of linear springs, the geometry renders the interactions nonlinear. This, in turn, leads to complex features of the straightforwardly realizable (in a mechanical experiment) system at hand. Such examples are manifested in the coherent structures of the system ranging from the familiar kinks to the more exotic rotobreathers 12] (or other structures such as domain walls or kinks with embedded defects [13]). They are also evident in the resonant energy transport features of the model that relate the energy conduction with the moving intrinsic localized modes (ILMs) that are present in the model (see below). We touch upon each of the above aspects to give a flavor of the rich and diverse properties that such a "geometrically-induced nonlinearity" model is endowed with. 


\section{MODEL}

The mechanical example that we examine consists of masses ("beads") that slide on fixed rings of radius $R$ (even though the radius can be variable from ring to ring, we will here consider it to be fixed). Furthermore, the centers of the rings are at distance $L$ between them. The chain of beads moving azimuthally along their respective rings is coupled through linear, elastic strings of a natural length $l_{0}$; see Fig. 1(a) for a schematic. There are two interesting subcases. The rings can be in the same plane or they can be in different planes. While the latter case is also of interest, we restrict ourselves to the former (coplanar rings) in what follows. Then,

$$
x_{n}=n L+R \cos \theta_{n}, \quad y_{n}=R \sin \left(\theta_{n}\right),
$$

and the distance between adjacent particles is given by:

$$
r_{n, n+1}=\sqrt{\left(x_{n+1}-x_{n}\right)^{2}+\left(y_{n+1}-y_{n}\right)^{2}} .
$$

The model Hamiltonian for linear, elastic interactions reads:

$$
H=\sum_{n}\left[\frac{1}{2} M R^{2}\left(\frac{\mathrm{d} \theta_{\mathrm{n}}}{\mathrm{dt}}\right)^{2}+\frac{1}{2} K\left(r_{n, n+1}-l_{0}\right)^{2}\right]
$$

Out of the 5 model parameters (particle mass $M$, spring constant $K$, disk radius $R$, distance between disk centers $L$ and string natural length $\left.l_{0}\right)$, two $(M, K)$ can be scaled out while out of the remaining three length scales, one can be used as a measure for the others (hence we set $R=$ 1 in what follows). The resulting equations of motion read:

$$
\begin{aligned}
\ddot{\theta}_{n} & =\left[\sin \left(\theta_{n+1}-\theta_{n}\right)-\sin \left(\theta_{n}-\theta_{n-1}\right)\right] \\
& +l_{0} L \sin \left(\theta_{n}\right)\left(\frac{1}{r_{n, n+1}}-\frac{1}{r_{n-1, n}}\right) \\
& -l_{0}\left(\frac{\sin \left(\theta_{n+1}-\theta_{n}\right)}{r_{n, n+1}}-\frac{\sin \left(\theta_{n}-\theta_{n-1}\right)}{r_{n-1, n}}\right),
\end{aligned}
$$

with $r_{n, n+1}^{2}=L^{2}+2-2 \cos \left(\theta_{n+1}-\theta_{n}\right)+2 L\left[\cos \left(\theta_{n+1}\right)-\right.$ $\left.\cos \left(\theta_{n}\right)\right]$. For convenience, we introduce the notation $L_{0}=\sqrt{L^{2}+4}$.

Ground States. The model of Eq. (4) supports numerous complex structures (a number of which we will examine below), but there are only three types of the ground state structures bearing a very simple form. The parameter space $\left(L, l_{0}\right)$ is naturally divided into three regions, depending on the corresponding types of ground state structure. We now summarize these structures (see also Fig. 11(b)).

For $l_{0}<L$, we are in regime I, where the lowest energy structure is

$$
\theta_{n}=\phi
$$

for constant $\phi$. Note that from static considerations, structures with any $\phi$ are in indifferent equilibrium, but in the presence of dynamic perturbations, only structures with $\phi=0, \pi$ are stable. The physical origin of the instability for $\phi \neq\{0, \pi\}$ can be easily understood considering the situation with all particles being at rest with $\theta_{n}=\phi$ and one particle oscillating with a finite amplitude near the equilibrium position. Taking into account higher order anharmonic terms, one can demonstrate an asymmetry in the torques acting on the particle with respect to deviation from the right versus deviation from the left. This asymmetry gives rise to the force driving the particle toward the closest stable position, namely $\phi=0$ or $\phi=\pi$, where the symmetry is restored. There are two more symmetric structures corresponding to $\phi=\pi / 2$ and $\phi=-\pi / 2$, but they are unstable. To illustrate this issue, for the chain of $N=400$ particles, we set the initial conditions $\dot{\theta}_{n}=0, \theta_{n}=\phi+r_{n}$, with different magnitudes of $\phi$ and $r_{n}$ being a random number homogeneously distributed on $[-0.05,0.05]$. In Fig. 1(c) we plot the time evolution of $\left\langle\theta_{n}\right\rangle=N^{-1} \sum_{n} \theta_{n}$. One can see that there are two stable positions $(\phi=0$ and $\phi=\pi)$ with respect to dynamic fluctuations.

We thus restrict our considerations to the stable equivalent structures, $\phi=0, \pi$. In this case, the dispersion relation reads:

$$
\omega(k)=2 \sqrt{1-\frac{l_{0}}{L}} \sin (\pi k) .
$$

In the limit $l_{0}=L$, the linear spectrum collapses to a single point (in this case $\omega=0$ ). Such geometrically induced limits where the linear spectrum collapses to a single point for all $k$ are an interesting feature of the present model (see below) and will be called purely anharmonic (PA) limits, as the harmonic linear part of the spectrum is eliminated in this case. This is a situation that bears some resemblence to the anti-continuum limit of models of ILMs [1].

Regime II is defined by $L<l_{0}<L_{0}$, where structures of the form:

$$
\theta_{n}= \pm\left[(-1)^{n} \phi+\delta \pi\right], \quad \sin ^{2} \phi=\frac{l_{0}^{2}-L^{2}}{4}
$$

have zero energy (all springs have their natural length); $\delta=0, \pm 1$. In this case, the linear spectrum is of the form:

$$
\omega(k)=\sqrt{l_{0}^{2}-L^{2}} \sqrt{L^{2}+\left(4-l_{0}^{2}\right) \sin ^{2}(\pi k)},
$$

which for $l_{0}=2$ becomes $k$-independent (PA limit), $\omega(k)=L \sqrt{4-L^{2}}$. The spectrum of Eq. (8) vanishes at $l_{0}=L$ which is the border between the type I and type II regimes.

Finally, regime III consists of the natural lengths such that $L_{0}<l_{0}$, when the ground state structure is

$$
\theta_{n}= \pm(-1)^{n} \frac{\pi}{2}
$$

In this case, the linear spectrum is given by

$$
\omega(k)=2 \sqrt{\left(1-\frac{4 l_{0}}{L_{0}^{3}}\right) \sin ^{2}\left(\frac{\pi}{2}-\pi k\right)+\frac{l_{0}}{L_{0}}-1 .}
$$


One can see that for $l_{0}=L_{0}^{3} / 4$, the width of linear spectrum vanishes (PA limit), $\omega(k)=L$.

Having discussed the ground states, we now give representative examples of the interesting nonlinear behavior that is possible in each of the regimes highlighted above.

\section{REGIME I}

Regime I supports an interesting rotobreather with one rotating particle. Such an example is shown in Fig. 22 a), where the particle $n=0$ rotates with angular velocity $\omega=20$ at $L=3$ and $l_{0}=1$. We show the stroboscopic picture of motion with intervals $T / 10$ (where $T$ is the period of the solution). Particles with positive and negative $n$ are practically at rest at their respective positions. The neighbors of $n=0$ particle can be at rest in the positions where the averaged (over a period) torque acting from the moving particle is equal to zero. For a large rotobreather frequency, the angular velocity of the $n=0$ particle is almost constant and, hence, the averaging over time can be substituted by averaging over angle $\theta_{0}$. Thus, an approximate rotobreather solution can be given as follows:

$$
\begin{gathered}
\theta_{n}=\omega t, \quad \text { for } n=0, \\
\theta_{n}= \pm \theta_{1}, \quad \text { for } n>0, \\
\theta_{n}= \pm\left(\pi-\theta_{1}\right), \quad \text { for } n<0,
\end{gathered}
$$

i.e., the 0th particle moves with constant angular velocity $\omega$ while all other particles are at rest at the positions defined through the angle $\theta_{1}$, which is a root of

$$
\int_{0}^{2 \pi} M_{1}\left(\theta_{0}, \theta_{1}\right) d \theta_{0}=0
$$

where $M_{1}\left(\theta_{0}, \theta_{1}\right)$ is the torque acting on the particle $n=$ 1 from the neighboring particles under the assumption that $\theta_{2}=\theta_{1}$ and $\theta_{0}$ is arbitrary. While the solutions of Eq. (12) with $\theta_{1}=0, \pi$ lead to unstable configurations, the root lying in the interval $\pi / 2<\theta_{1}<\pi$ leads to a stable rotobreather which very accurately captures our numerical findings (see the caption of Fig. 2(a)).

Note that in the case of roto-breather presented in Fig. 2(a), dynamic fluctuations are absent. Introduction of such fluctuations would result in the appearance of a weak forces driving the particles with $n<0$ to the position $\theta_{n}=0$ and particles with $n>0$ to the positions with $\theta_{n}=\pi$ [see Fig. प(c)]. An equilibrium state in this case would look similar to what is presented in Fig. 2(a) only in the vicinity of the roto-breather, but more distant particles would approach the positions which are stable against dynamic fluctuations.

\section{REGIME II}

As two prototypical examples of the structures that can be supported in regime II, we derive a kink solution and provide a numerical example of a highly localized, moving ILM.

Kink solution. Using

$$
\theta_{n}=(-1)^{n}\left(\frac{\pi}{2}+\varepsilon_{n}\right),
$$

where $\varepsilon_{n} \ll \pi$ and assuming that $\varepsilon_{n}$ varies slowly with $n$, we obtain from Eq. (44) [up to cubic terms]

$$
\begin{aligned}
\ddot{\varepsilon}_{n} & =\frac{B}{L^{2}}\left(\varepsilon_{n-1}-2 \varepsilon_{n}+\varepsilon_{n+1}\right)+C \varepsilon_{n} \\
& -\frac{D}{16}\left[\left(\varepsilon_{n+1}+\varepsilon_{n}\right)^{3}+\left(\varepsilon_{n}+\varepsilon_{n-1}\right)^{3}\right],
\end{aligned}
$$

with $B=L^{2}\left(1-\frac{4 l_{0}}{L_{0}^{3}}\right), C=4\left(1-\frac{l_{0}}{L_{0}}\right)$ and $D=$ $\frac{8}{3}\left(1-\frac{l_{0}\left(L^{2}+1\right)}{L_{0}^{3}}\right)$. In the continuum limit, Eq. (14) reduces to the $\varphi^{4}$-equation,

$$
\varepsilon_{t t}=B \varepsilon_{x x}+C \varepsilon-D \varepsilon^{3} .
$$

When $C>0$ and $D>0$, the background potential has a double-well structure and Eq. (15) supports topological solitons (kinks and antikinks) 14]. When the kink width is much greater than $L$, the solution of Eq. (15) can be used to approximate the kink solution of the discrete Eq. (4):

$$
\theta_{n}=(-1)^{n}\left[\frac{\pi}{2} \pm \sqrt{\frac{B}{D}} \tanh [Q(n L-v t)]\right]
$$

where $v<\sqrt{B}$ is the kink velocity and $Q=$ $\sqrt{C /\left[2\left(v^{2}-B\right)\right]}$. We have verified that even for relatively small kink widths (i.e., moderate discreteness), Eq. (16) approximates well the numerically obtained kink solutions [see Fig. 2(b)].

We note in passing that in regime II there exist various different types of domains, as illustrated in Eq. (7). Hence, there are possibilities to create additional kinks, connecting different steady states than the ones presented above.

Moving ILM. An interesting example of a moving ILM is presented in Fig. [ 3 for $l_{0}=2$ (PA limit), $L=1.2$. The localized mode emerged from the local perturbation introduced to $n=0$ particle of the structure $\theta_{n}=(-1)^{n} \phi$ by setting $\theta_{0}=\phi+\pi / 2$ with zero initial velocities for all particles at $t=0$. The asymmetry in the displacements of particles renders this structure less tractable analytically. We will give a very simple analytical approximation for the ILMs (standing and moving) in regime III where the displacements are symmetric. However, we believe that the mechanism of ILM propagation in the regime II is the same as in the regime III, i.e., the relay-like resonant energy exchange between particles.

\section{REGIME III}

Standing and moving ILMs. Regime III supports ILMs which, depending on model parameters, can be moving 
or standing. We have also found that the existence of standing ILMs precludes the existence of moving ones and vice versa. To better understand this phenomenon we first solve an auxiliary problem of resonant energy transfer and then give some simple analytical estimates for standing and moving ILMs. The robustness of moving ILMs against their collisions is also verified numerically.

Here we consider the PA limit, $l_{0}=L_{0}^{3} / 4$. If a particle is forced according to:

$$
\theta_{0}(t)=\frac{\pi}{2}+A \sin (\omega t)
$$

with $A \ll \pi$, we are interested in the motion of its nearest neighbors, $\theta_{-1}(t)=\theta_{1}(t)=-\frac{\pi}{2}-\varepsilon(t)$ with $\varepsilon \ll \pi$, assuming that all other particles are at rest at their equilibrium positions. Retaining up to linear terms in $\varepsilon$ and cubic in $A$, we obtain

$$
\begin{array}{r}
\ddot{\varepsilon}+\omega_{0}^{2} \varepsilon=\beta A^{2} \sin ^{2}(\omega t)+\gamma A^{3} \sin ^{3}(\omega t), \\
\omega_{0}=L, \quad \beta=\frac{L\left(2-L^{2}\right)}{4 L_{0}^{2}}, \quad \gamma=\frac{2-L^{2}+2 L^{4}}{3 L_{0}^{4}}-\frac{1}{6},
\end{array}
$$

with the particular solution

$$
\begin{array}{r}
\varepsilon(t)=\frac{\beta A^{2}}{2 \omega_{0}^{2}}+\frac{3 \gamma A^{3}}{4\left(\omega_{0}^{2}-\omega^{2}\right)} \sin (\omega t) \\
-\frac{\beta A^{2}}{2\left(\omega_{0}^{2}-4 \omega^{2}\right)} \cos (2 \omega t)-\frac{\gamma A^{3}}{4\left(\omega_{0}^{2}-9 \omega^{2}\right)} \sin (3 \omega t),
\end{array}
$$

which gives the first three resonance harmonics. When the forced particle oscillates with a nearly resonant frequency, the amplitude of its neighbors can grow significantly.

Equation (18) does not take anharmonicity into account. The latter effect was studied numerically, where we found that the anharmonicity is hard, i.e., the oscillation frequency of the $n=1$ particle grows as a function of amplitude, for $L<L^{*} \approx 1.65$, and the situation is reversed for $L>L^{*}$.

The dynamics of the chain with one forced particle differs qualitatively for hard and soft anharmonicity. For $L<L^{*}$, when the free particle has maximum amplitude, it oscillates in phase with the forced particle, and its amplitude excceeds $A$ in the resonance regime while for $L>L^{*}$ it does not because the particles oscillate out of phase. In other words, efficient inter-particle energy exchange occurs only for hard anharmonicities. For the chain with soft anharmonicity, any local perturbation remains local.

We carry out the following numerical experiments. We excite a single site according to Eq. (17) for times $0 \leq$ $t \leq \tau$ with $\tau=5000$ and calculate the power of the energy source, $W=E / \tau$, where $E$ is the total energy of the chain at $t=\tau$. The chain is long enough so that at $t=\tau$ the perturbation produced by the forced particle has not reached the boundaries.

We have found that $W$ can be nonzero only for $L<L^{*}$, regardless of the magnitudes of $A$ and $\omega$ in Eq. (17). The mechanism of the energy transfer is the emission of mov-

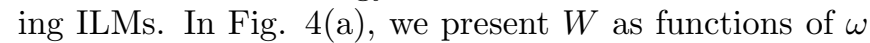
for $L=1$ and for $A=0.2,0.3,0.4$. It can be seen that the smaller $A$ is, the narrower the window of $W>0$. The figure also shows the distribution of energy in the chain at $t=\tau$ for $L=1, l_{0}=L_{0}^{3} / 4$, and driving parameters $A=0.4$ and (b) $\omega=0.992$, (c) $\omega=1.020$, (d) $\omega=1.033$. In (b) and (d) (edges of the window with $W>0$ ), the forced particle emits ILMs periodically while in (c) (central part of the window) chaotically. For $L>L^{*}$, there is no efficient energy exchange between particles and moving ILMs are not possible. Instead, stable standing ILMs arise that are localised at the excited particle. An approximate solution can be expressed by a conventional perturbation method assuming that only one particle moves:

$$
\begin{array}{r}
\theta_{0}(t)=\frac{\pi}{2}+A_{1} \sin (\omega t)+A_{3} \sin (3 \omega t), \\
\omega^{2}=\omega_{0}^{2}+\frac{3}{4} B A_{1}^{2}, \quad A_{3}=\frac{-B A_{1}^{3}}{32 \omega_{0}^{2}+27 B A_{1}^{2}},
\end{array}
$$

where $\omega_{0}^{2}=\frac{4 l_{0}\left(L^{2}+2\right)}{L_{0}^{3}}-2, B=\frac{1}{3}-l_{0} \frac{5 L^{6}+8 L^{4}+16 L^{2}+16}{3 L_{0}^{7}}$.

The moving ILM is (practically) localized at three particles:

$$
\begin{gathered}
\theta_{n-1}=a A_{1} \sin \left(\omega t \pm \frac{2 \pi}{3}\right), \\
\theta_{n}=A_{1} \sin (\omega t)+A_{3} \sin (3 \omega t), \\
\theta_{n+1}=a A_{1} \sin \left(\omega t \mp \frac{2 \pi}{3}\right),
\end{gathered}
$$

where upper and lower signs correspond to ILM moving in positive and negative directions, respectively. The amplitude $A_{1}$ of the ILM is a free parameter. The relation between $A_{1}$, the ILM frequency $\omega$, and the third harmonic amplitude, $A_{3}$, is given by Eq. (20). Empirically we have found that $a=0.4$ gives a good result over a wide range of ILM amplitudes (see Fig. [5).

In Fig. 6] we show an in-phase collision of two ILM defined by Eq. (21) with $A=0.7, a=0.4$. Model parameters are $L=1, l_{0}=L_{0}^{3} / 4$ (PA limit). Shown are the snapshots of $H_{n}$ at different time $t$, where $H_{n}$ is the total energy of $n$th particle (kinetic and potential). We can see that such moving ILMs can interact with each other in a quasi-elastic fashion.

Rotobreathers. Regime III also supports a rotobreather with one rotating particle. Here again, as in Sec. III we assume that for a rotobreather with sufficiently large frequency the torque acting from the rotating particle on its nearest neighbors can be estimated by averaging over angle. In Fig. [7 we show the (averaged over angle) torque acting from rotating $(n=0)$ particle on its nearest neighbor $(n=1)$, which is assumed to be at rest at $\theta_{1}$, for $l_{0}=L_{0}^{3} / 4$ (PA limit) and $L=2$ (dotted), $L=1.5$ (dashed), $L=1$ (solid). We are interested in the positions $\theta_{1}$ corresponding to zero torque. For $L<1.3$ there are only two roots (one of them is stable) and for 
$L>1.3$ there are four roots (two of them are stable). For $L=5$, for example, the stable root was found at $\theta_{1}=-\pi / 2+0.084$ which is in a good agreement with what is observed for the rotobreather presented in Fig. 8 For $L=5$, another stable root for the $n=1$ particle was found at $\theta_{ \pm} 1=\pi / 2+0.135$ and the existance of this rotobreather was also confirmed numerically (see Fig. 9).

\section{CONCLUSIONS}

In this paper we have presented a novel nonlinear dynamical system, consisting of an easily realizable mechanical example where the nonlinearity is induced by the geometry of the problem. We have illustrated the laws of motion and the rich static, dynamic (both equilibrium and non-equilibrium) behavior of the system. We have identified some of the relevant coherent structures including kink-like heteroclinic connections and roto-breathing periodic orbits and have seen some of the interesting dynamical phenomenology including the "conducting" (for hard anharmonicities) or "insulating" (for soft anharmonicities) behavior of the system and the role of moving or standing ILMs, respectively, as energy carriers. It would be of interest to examine further from an analytical (as well as from a numerical or experimental) perspective the phenomenological wealth of such a model. Such studies are currently in progress and will be reported in future publications.
[1] S. Aubry, Physica 103D, 201 (1997); S. Flach and C. R. Willis, Phys. Rep. 295, 181 (1998); Physica 119D, (1999); P.G. Kevrekidis, K.Ø. Rasmussen and A.R. Bishop, Int. J. Mod. Phys. B 15, 2833 (2001); see also A. J. Sievers and S. Takeno, Phys. Rev. Lett. 61, 970 (1988); S. Takeno and S. Homma, Prog. Theor. Phys. 70, 308 (1983).

[2] G. Kopidakis, S. Aubry and G. P. Tsironis, Phys. Rev. Lett. 87, 165501 (2001).

[3] H. E. Nistazakis, P. G. Kevrekidis, B. A. Malomed, D. J. Frantzeskakis and A. R. Bishop, Phys. Rev. E 66, 015601(R) (2002).

[4] S. Lepri, R. Livi and A. Politi, Phys. Rep. 377, 1 (2003).

[5] E. C. Aifantis, Int. J. Nonlinear Mechanics 31, 797 (1996); E. C. Aifantis, in: The Mechanics of Dislocations, eds. E. C. Aifantis and J. P. Hirth, pp. 127-146, ASM, Metals Park, 1985.

[6] Y. B. Gaididei, S. F. Mingaleev and P. L. Christiansen, Phys. Rev. E 62, R53 (2000).

[7] P. G. Kevrekidis, B. A. Malomed and A. R. Bishop, Phys. Rev. E 66, 046621 (2002).

[8] P. G. Kevrekidis, V. V. Konotop, B. A. Malomed, and
A. R. Bishop Phys. Rev. E 70, 047602 (2004).

[9] I. Bena, A. Saxena and J. M. Sancho, Phys. Rev. E 66, 036617 (2002); I. Bena, A. Saxena, G. P. Tsironis, M. Ibanes and J. M. Sancho, Phys. Rev. E 67, 037601 (2003).

[10] G. P. Tsironis, M. Ibanez, and J. M. Sancho, Europhys. Lett. 57, 697 (2002); M. Ibanes, J. M. Sancho and G. P. Tsironis, Phys. Rev. E 65, 041902 (2002).

[11] J. F. R. Archilla, P. L. Christiansen, S. F. Mingaleev and Yu. B. Gaididei, J. Phys. A: Math. and Gen. 34, 6363 (2001); J. F. R. Archilla, P. L. Christiansen, and Yu. B. Gaididei, Phys. Rev. E 65, 016609 (2001); J. Cuevas, J. F. R. Archilla, Yu. B. Gaididei and F. R. Romero, Physica D 163, 106 (2002); S. Homma and S. Takeno, Prog. Theor. Phys. 72, 679 (1984).

[12] S. Takeno and M. Peyrard, Physica D92, 140 ( 1996); Phys. Rev. E 55, 1922 (1997).

[13] S. V. Dmitriev et al. (unpublished).

[14] T. I. Belova and A. E. Kudryavtsev, Physics-Uspekhi 40, 359 (1997). 

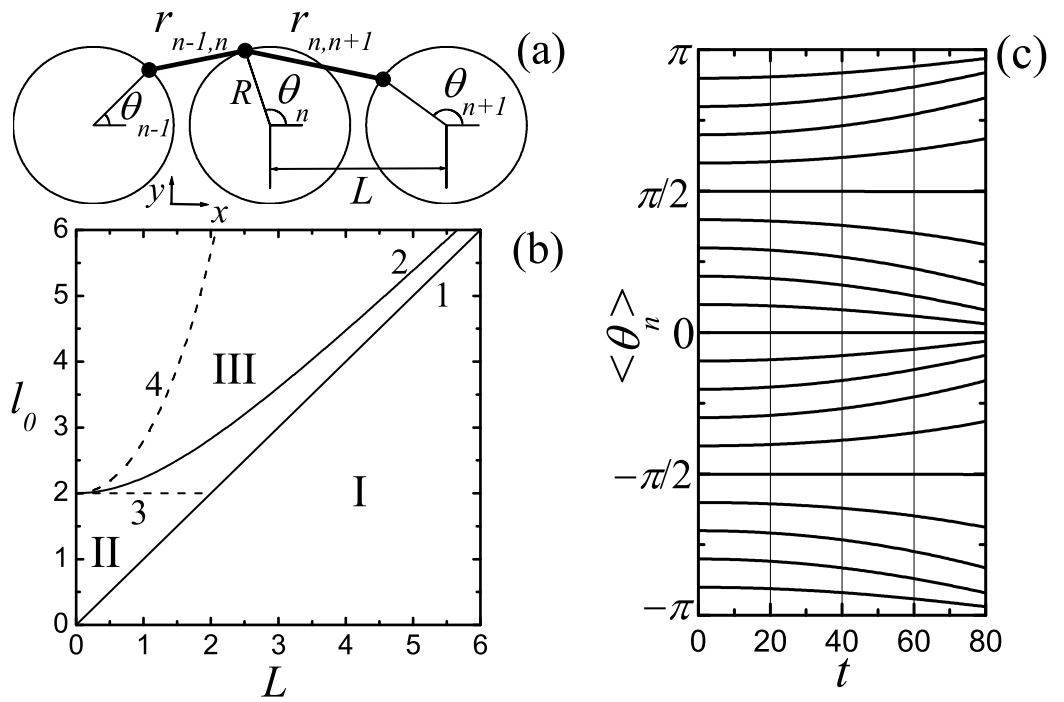

FIG. 1: (a) The bead-ring configuration: the rings of radii $R$ are at distance $L$ between them (either on the same or on different planes). The dynamical variable of interest for each particle is its azimuthal angle $\theta_{n}$ and the chain has a nearest neighbor coupling through linear, elastic springs of natural length $l_{0}$. The distance between adjacent particles is denoted by $r_{n, n+1}$. (b) The parameter space of the model $\left(L, l_{0}\right)$ divided into three regions with different ground state structures. (c) Illustration of dynamical instability in the regime I for all $\phi$ except for $\phi=0$ and $\phi= \pm \pi$. Average atomic positions $<\theta_{n}>$ for the chain of $N=400$ particles are shown as the functions of time. Initially the particles are placed at $\theta_{n}=\phi$ with different magnitudes of $\phi$, and a small amplitude random perturbation is introduced in the particle positions to initiate their vibrations. 

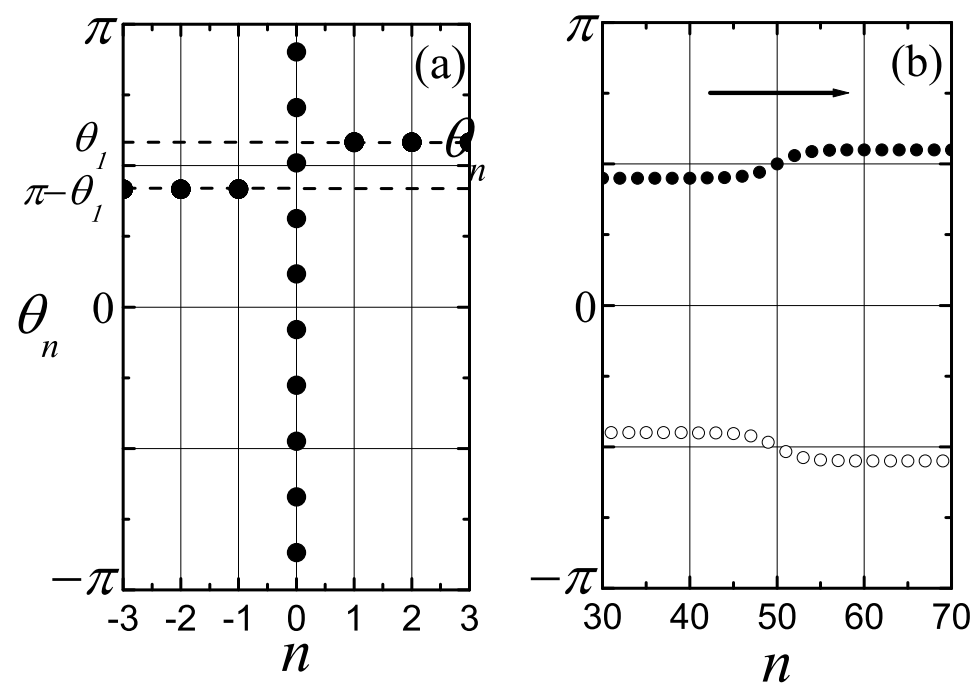

FIG. 2: (a) Numerical solution for a rotobreather in regime I. The $n=0$ particle rotates with angular velocity $\omega=20$ at $L=3$ and $l_{0}=1$. We show the stroboscopic picture of motion with intervals $T / 10$ (a tenth of the period $T$ ). Particles with positive and negative $n$ are practically at rest at $\theta_{n}=\pi / 2 \pm$ 0.261. Numerically we found the root of Eq. (12) at $\theta_{1}=$ $\pi / 2+0.259$. (b) A kink solution is shown in regime II for $v=0.2, L=1, l_{0}=\sqrt{4 \sin ^{2} \phi+L^{2}}$, with $\phi=(9 / 20) \pi$ (close to $\phi=\pi / 2)$. Even and odd particles are shown by filled and open circles, respectively. Even in this case of (not very big) kink width, Eq. (16) provides a very good approximation. The arrow shows the direction of propagation of the kink.

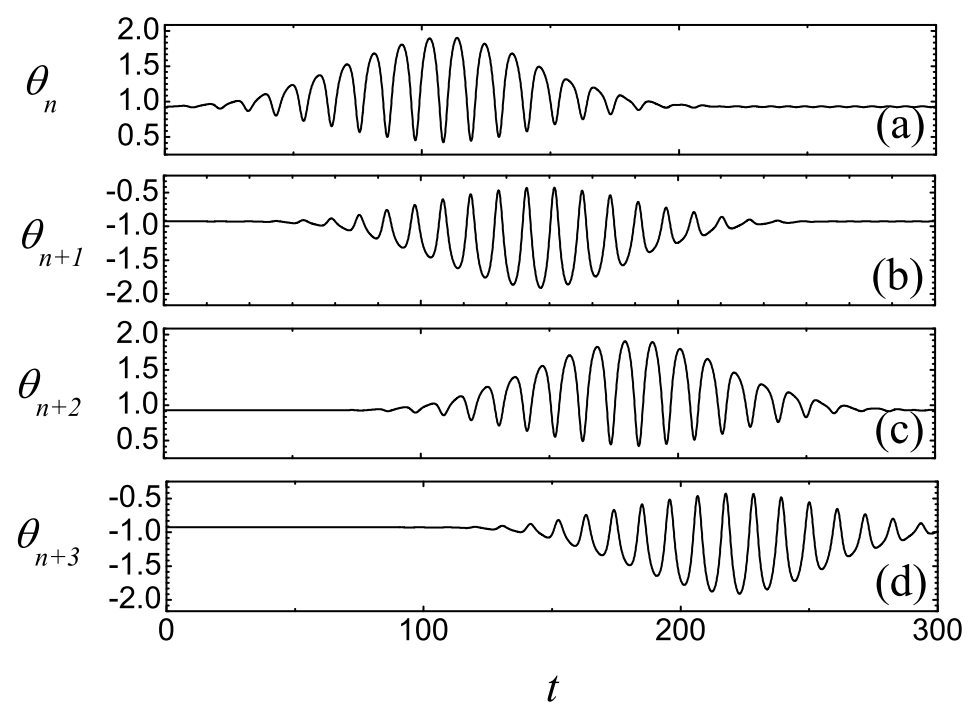

FIG. 3: Moving ILM in the regime II for $l_{0}=2$ (PA limit) and $L=1.2$. 


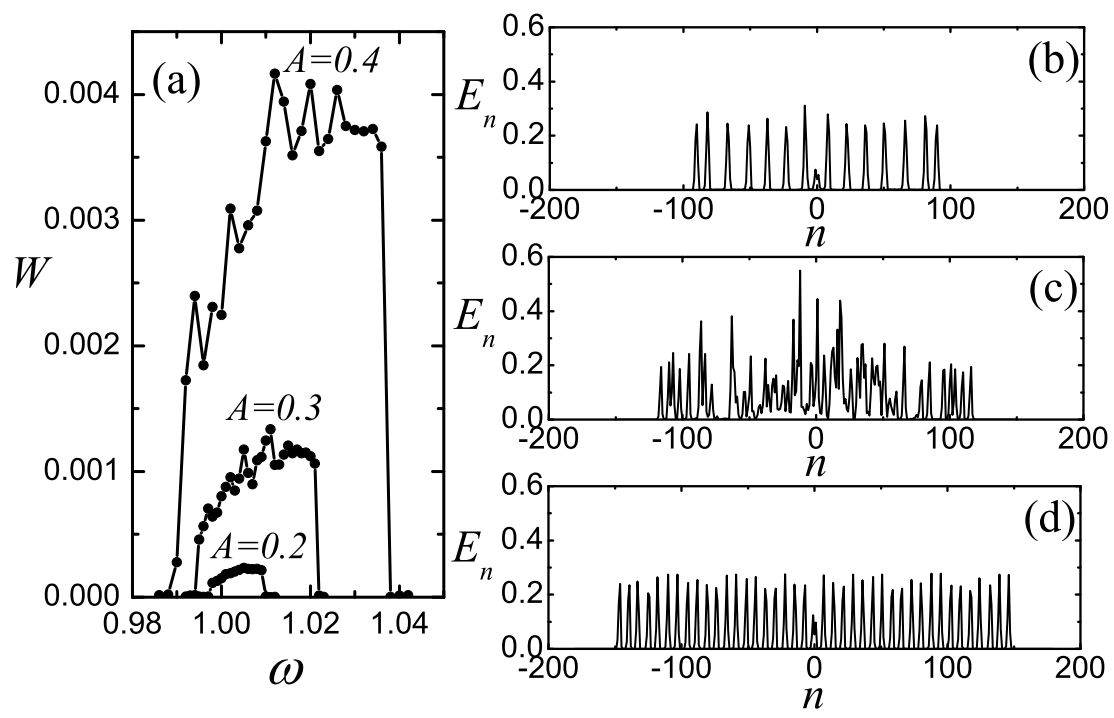

FIG. 4: (a) The power of the energy source $W$ is shown as a function of the driving frequency $\omega$ for $L=1$ and for $A=$ $0.2,0.3,0.4$. Right Panel: the distribution of energy at $t=\tau$. Model parameters are $L=1, l_{0}=L_{0}^{3} / 4, A=0.4$ and (b) $\omega=0.992$, (c) $\omega=1.020$, (d) $\omega=1.033$. The panel shows the particle energies $E_{n}$, averaged over the period $2 \pi / \omega$. In (b) and (d) the forced particle emits ILMs periodically, while in (c) it emits chaotically. 

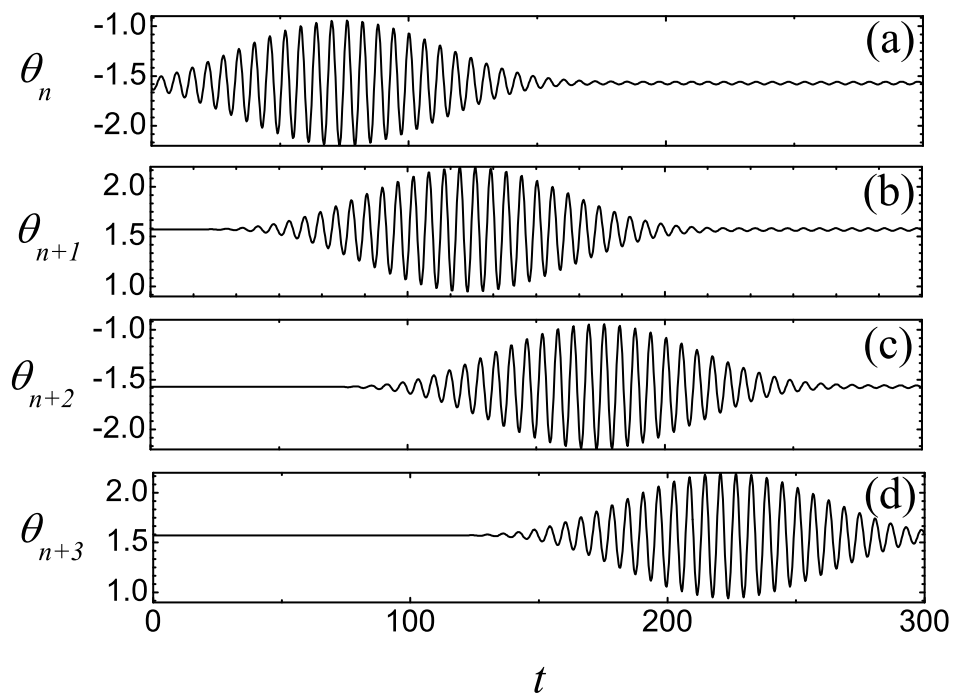

FIG. 5: A moving ILM is shown by the functions $\theta_{n}(t)$ for the four nearest nodes. Particles show the relay-like motion oscillating near the equilibrium positions $\theta_{n}=(-1)^{n}(\pi / 2)$. Model parameters are $l_{0}=L_{0}^{3} / 4$ (PA limit in regime III), and $L=1\left(<L^{*}\right)$. The parameters in the solution Eq. (21) are $A_{1}=0.6, a=0.4$. Marginal radiation can be seen after the ILM passes a node (at large times). The ILM propagates rather slowly, it travels one lattice spacing $L$ in about $8 T$.

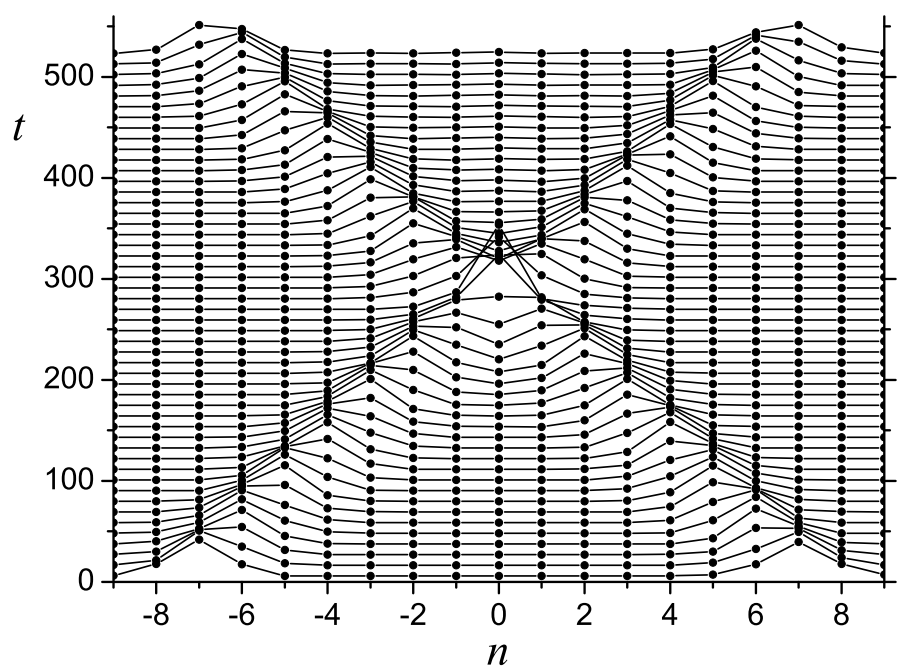

FIG. 6: Elasticity of the in-phase collision of two moving ILMs defined by Eq. (21) with $A=0.7, a=0.4$. The model parameters are $L=1\left(<L^{*}\right)$ and $l_{0}=L_{0}^{3} / 4$ (PA limit). 


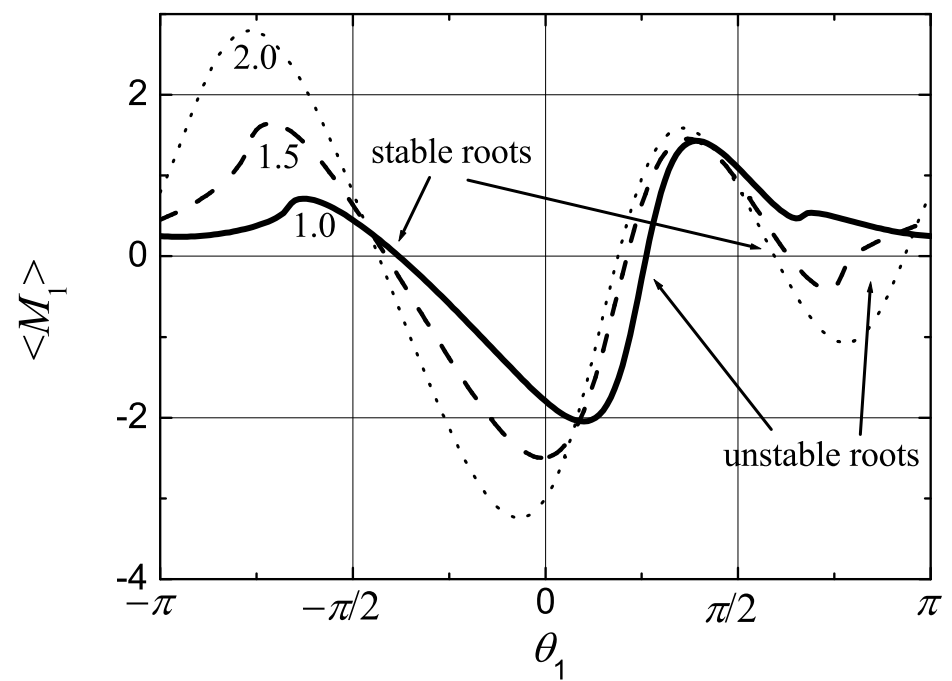

FIG. 7: Torque (averaged over the angle $\theta_{0}$ ) acting from rotating particle $(n=0)$ on its nearest neighbor $(n=1)$ which is at rest at $\theta_{1}$ for $l_{0}=L_{0}^{3} / 4$ (PA limit) and $L=2$ (dotted), $L=1.5$ (dashed), $L=1$ (solid).
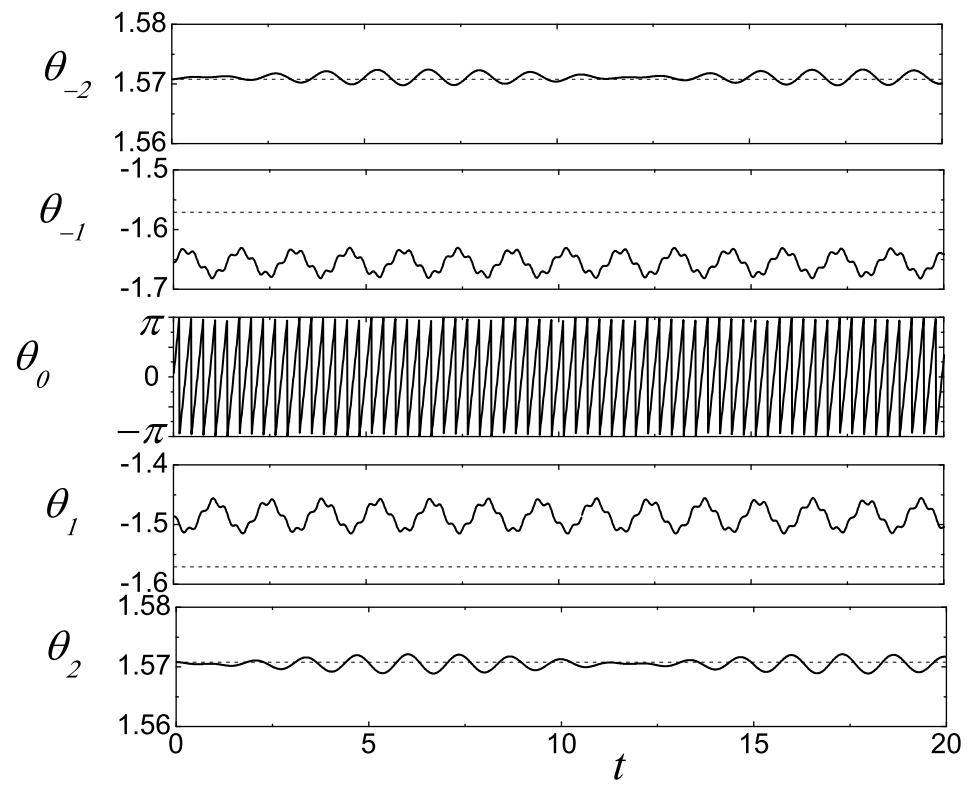

FIG. 8: Rotobreather in the regime III initiated by setting initial angular velocity $\dot{\theta}_{0}=20$ and initial positions of the $n= \pm 1$ particles $\theta_{ \pm} 1=-\pi / 2 \pm 0.084$ corresponding to zero averaged torque acting from $n=0$ particle. Model parameters are $L=5$ and $l_{0}=L_{0}^{3} / 4$ (PA limit). Dashed horizontal lines show $\theta_{n}= \pm \pi / 2$. 


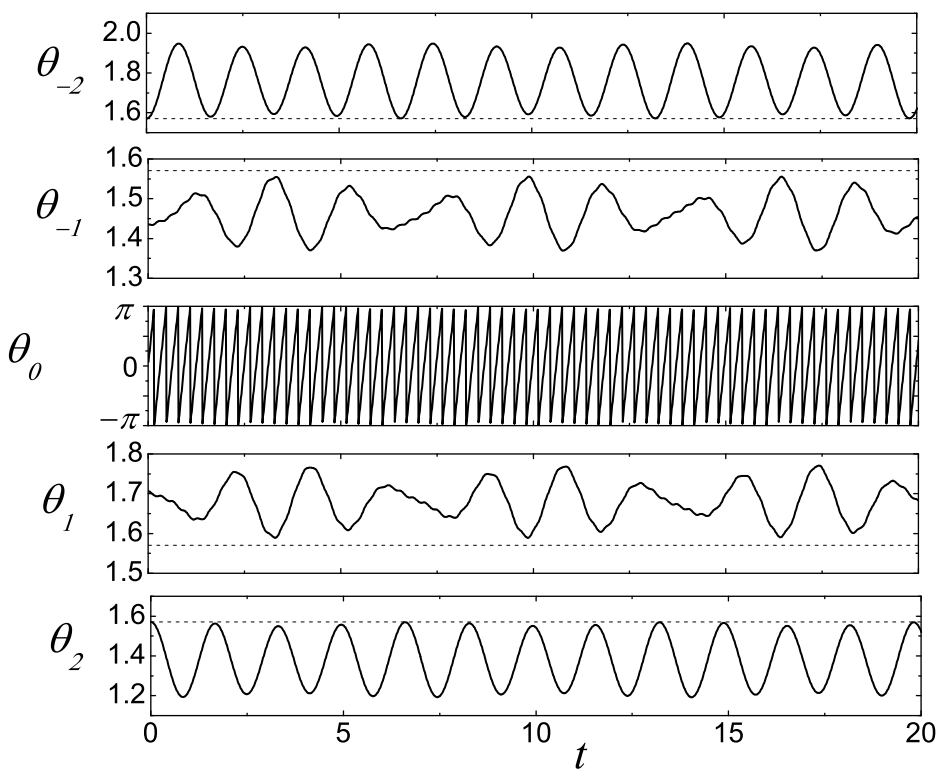

FIG. 9: Rotobreather corresponding to the root of equation $<M_{1}>=0$ which appeares at large $L$. In this case $L=5$, $l_{0}=L_{0}^{3} / 4$ (PA limit) and the root was found for $n= \pm 1$ particles at $\pi / 2 \pm 0.135$. Note that here the particles $n= \pm 1$ oscillate near $\pi / 2$ but not near $-\pi / 2$ as in the case presented in Fig. 8 This is because the stable root which appeares at $L>1.3$ is shifted by, roughly, $\pi$ compared to the root existing for all $L$ (see Fig. 17). 